\title{
Sudden Collapse of a Granular Cluster
}

\author{
Devaraj van der Meer, Ko van der Weele, and Detlef Lohse \\ Department of Applied Physics and J. M. Burgers Centre for Fluid Dynamics, University of Twente, \\ P.O. Box 217, 7500 AE Enschede, The Netherlands
}

(Received 6 July 2001; revised manuscript received 26 November 2001; published 15 April 2002)

Single clusters in a vibro-fluidized granular gas in $N$ connected compartments become unstable at strong shaking. They are experimentally shown to collapse very abruptly. The observed cluster lifetime (as a function of the driving intensity) is analytically calculated within a flux model, making use of the self-similarity of the process. After collapse, the cluster diffuses out into the uniform distribution in a self-similar way, with an anomalous diffusion exponent $1 / 3$.

DOI: $10.1103 /$ PhysRevLett.88.174302

One of the key features of a granular gas, making it fundamentally different from ordinary molecular gases, is its tendency to spontaneously separate into dense and dilute regions [1]. This clustering originates from the dissipative nature of the particle collisions. It is an unwanted effect in many applications where granular material is brought into motion. Therefore we study (within a simple geometry) how declustering occurs. We find that the breakdown of a cluster can be very abrupt, making declustering very different from clustering in reverse time order.

The experimental system (see Fig. 1) consists of a row of $N$ equal compartments, separated by walls of height $h=25.0 \mathrm{~mm}$ and filled with a few hundred steel beads of diameter $3.0 \mathrm{~mm}$. We start out with all the particles in the middle compartment and bring them into a gaseous state by shaking the system vertically. For weak shaking the cluster is stable: after some initial spilling, a dynamical equilibrium is established between the outflux of slow particles from the cluster and the influx of fast particles from outside [2-4]. For sufficiently strong shaking, however, the cluster breaks down.

Two different regimes are observed: (i) At very strong shaking the breakdown occurs immediately, and the cluster spreads out over the boxes with its profile widening as $t^{1 / 3}$ (instead of the standard $t^{1 / 2}$ diffusion law). (ii) At less violent shaking, the cluster seems to remain stable for a long time, showing only a small leakage to its neighbors. But suddenly it collapses and subsequently diffuses over all boxes. The sudden death of the cluster is depicted in Fig. 1.

One thing this figure shows is that the breakdown of a cluster is strikingly different from the reverse process of cluster formation, which is known to take place gradually and (for all $N \geq 3$ ) via transient states showing clusters in several boxes $[3,4]$. This lack of time reversibility is yet another consequence of the dissipation in the system.

The abruptness of the collapse allows us to define a cluster lifetime $\tau$ [via $\ddot{n}_{c l}(\tau)=0$; see Fig. 1e]. In Fig. 2 the measured lifetimes are plotted as a function of the inverse shaking strength $\tilde{B}$ [Eq. (2)] for various values of $N$. The data lie on a universal envelope curve, until at some critical value $\tilde{B}_{c, N}$ (which grows with $N$ ) they diverge.
PACS numbers: 45.70.-n, 02.60.Lj, 05.45.-a

All of the above experimental observations can be accounted for quantitatively by means of the flux model of Refs. [2-4]. At the heart of this model is a flux function $F\left(n_{k}\right)$, describing the outflow from the $k$ th box to each of its neighbors. It is a nonmonotonic function of $n_{k}$ (the particle fraction in the box): $F\left(n_{k}\right)$ first increases with $n_{k}$, but beyond a certain value of $n_{k}$ it decreases again, as the increasingly frequent inelastic collisions slow the particles down, so that they cannot make it over the wall to the neighboring compartments anymore. The precise form of $F\left(n_{k}\right)$ is not very critical, as long as it is a one-humped function. We will use Eggers' flux function [2]

$$
F\left(n_{k}\right)=C \sqrt{\tilde{B}} n_{k}^{2} e^{-\tilde{B} n_{k}^{2}},
$$

with

$$
\tilde{B} \propto \frac{g h r^{4}(1-e)^{2} P^{2}}{S^{2}(a f)^{2}} .
$$

The driving parameter $\tilde{B}$ depends on the total number of particles $P$ and their properties (radius $r$, restitution coefficient $e$ of the particle collisions), on the geometry of the system (height $h$ of the walls, ground area $S$ of each box), and on the frequency $f$ and amplitude $a$ of the

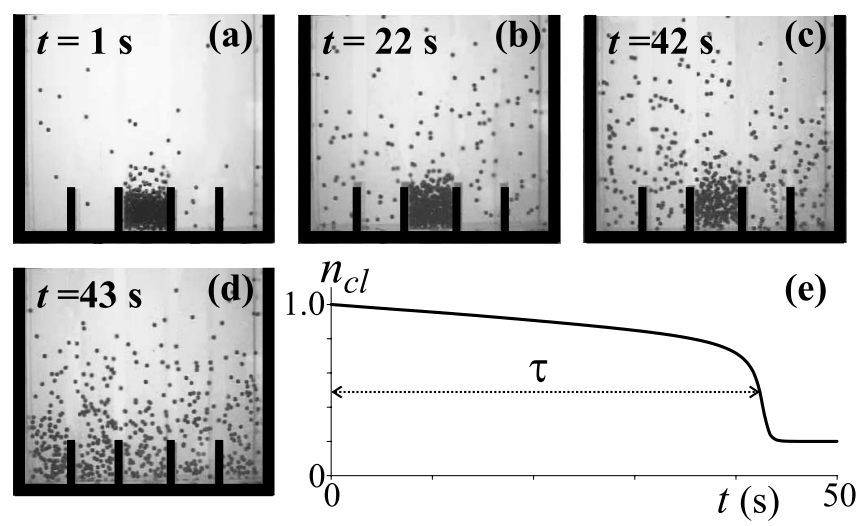

FIG. 1. (a)-(d) Four images from a five-box experiment, at driving parameter $\tilde{B}=8.2$. The cluster is clearly present until $t=42 \mathrm{~s}$, then suddenly collapses, leaving no trace one second later. (e) The time evolution of the cluster fraction $n_{c l}(t)$, evaluated from the flux model. 


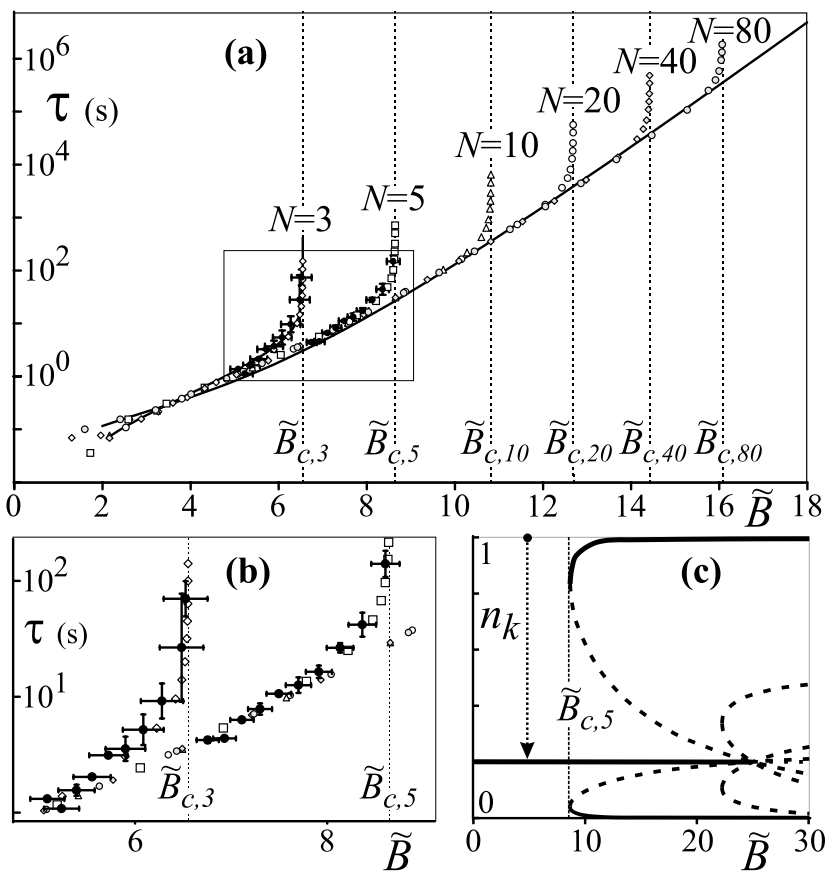

FIG. 2. (a) Lifetime $\tau$ vs driving parameter $\tilde{B}$, determined from experiments with $N=3,5$ boxes (dots with error bars), and from numerical evaluation of the flux model with $N=$ $3,5,10,20,40,80$ boxes (empty symbols). The solid curves are analytical solutions for $N=3$ [9] and for the envelope curve, which goes roughly as $\exp ((3 / 2) \tilde{B})$ [cf. Eq. (13)]. (b) Enlargement of (a), showing the experimental results for $N=3$ and 5 in more detail. Every point is based on 15 repetitions of the experiment; the vertical error bars denote the maximal deviation from the average $\tau$ measured, the horizontal ones represent the accuracy in $\tilde{B}$. (c) Bifurcation diagram for $N=5$, showing the critical value $\tilde{B}_{c, 5}$. Declustering occurs to the left of this value.

shaking. The factor $C$ determines only the absolute rate of the flux, and can be incorporated in the time scale.

The equation of motion for the fraction in box $k$ is [5]

$$
\frac{d n_{k}}{d t}=F\left(n_{k-1}\right)-2 F\left(n_{k}\right)+F\left(n_{k+1}\right)
$$

where $k=1,2, \ldots, N$. Here we assume a nearest neighbor interaction, and a cyclic arrangement of the boxes $(k=$ $N+1$ equals $k=1$ ). We further impose particle conservation, $\sum_{k} n_{k}=1$.

The numerical results shown in Figs. 1e and 2 have been obtained using the above flux model, starting out with all particles in one box (labeled $c l$ ). They quantitatively agree with the experimental observations. The decaying cluster goes through three different stages.

The starting stage is a very short one, in which both $n_{c l}$ and $F\left(n_{c l}\right)$ display a jump compared to $n_{i}$ and $F\left(n_{i}\right)$ in the surrounding boxes, $i=1,2, \ldots$ (we have to consider one side only because of the symmetry in the system).

In the second stage, the flux has become continuous but the particle fraction remains discontinuous. However, its low-density counterpart $n_{0}$ [defined by $F\left(n_{0}\right)=F\left(n_{c l}\right)$ ] does continuously connect to $n_{1}$. We will use this fact later in the analysis of the envelope curve. The flux gradually grows, and eventually $F\left(n_{c l}\right)$ reaches its maximum value. This is accompanied by rapid density changes and the sudden death of the cluster at the lifetime $\tau$.

In the third and last stage, both $n_{c l}$ and $F\left(n_{c l}\right)$ fit continuously to the other boxes (see Fig. 3a). The remains of the cluster diffuse over the whole system until the uniform distribution is reached.

In what follows we will analytically solve the flux model. First we focus on the third stage. We rewrite the problem into its continuum version, by setting $n(x, t) \equiv$ $n_{k}(t)(x=k w$ by definition, where the box width $w$ will be incorporated in the $x$ scale). Equation (3) then becomes

$$
\begin{aligned}
\partial_{t} n & =\partial_{x x} F(n(x, t)) \\
& =C \sqrt{\tilde{B}} \partial_{x x}\left(n(x, t)^{2} e^{-\tilde{B} n(x, t)^{2}}\right),
\end{aligned}
$$

and the conservation condition takes the form $\int_{-\infty}^{\infty} n(x, t) d x=1$.

For very strong shaking [regime (i), where $\tau$ is vanishingly small] the diffusive stage sets in almost immediately. Here $\tilde{B} \rightarrow 0$, and Eq. (4) reduces to

$$
\partial_{t} n=C \sqrt{\tilde{B}} \partial_{x x}\left(n^{2}\right)=2 C \sqrt{\tilde{B}}\left[\left(\partial_{x} n\right)^{2}+n \partial_{x x} n\right],
$$

which is known as the porous media equation [6,7]. The decay of the cluster in this limit is depicted in Fig. 3a. It is self-similar: all curves in Fig. 3a fall onto a single curve if we properly rescale the axes (Fig. 3b). The original partial differential equation (PDE) can thus be brought back to an ordinary differential equation (ODE) in terms of the self-similarity variable $\eta=x /\left(C \tilde{B}^{1 / 2} t\right)^{1 / 3}$. With $n(x, t)=H(\eta) /\left(C \tilde{B}^{1 / 2} t\right)^{1 / 3}$, Eq. (5) now takes the form

$$
\partial_{\eta \eta}\left(H^{2}\right)+\frac{1}{3} \partial_{\eta}(\eta H)=0 .
$$

Its symmetric solution is $H(\eta)=H_{0}-(1 / 12) \eta^{2}$ [with the constant $H_{0}=\left(3^{1 / 3}\right) / 4 \approx 0.361$ determined by $\left.\int_{-\infty}^{\infty} H(\eta) d \eta=1\right]$. This inverted parabola, depicted in Fig. 3b, represents in one curve all the stages of Fig. 3a. The scaling of the axes shows that the height of the cluster decreases as $t^{-1 / 3}$, and its width grows as $t^{1 / 3}$. This anomalous diffusion (with exponent $1 / 3$ ) is also found in porous media [6,7]. The slowed down diffusion of the
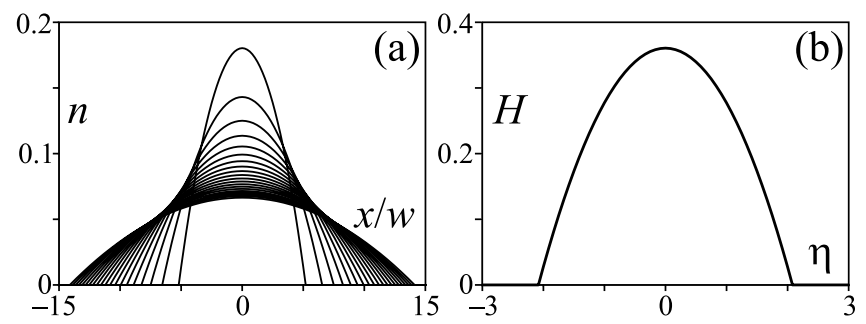

FIG. 3. (a) The diffusing profile at successive times $t$ (in the limit $\tilde{B}=0$ ). (b) The function $H(\eta)$ onto which all the curves in plot (a) collapse by a proper rescaling of the axes $\left(H \propto n t^{1 / 3}\right.$ and $\left.\eta \propto x t^{-1 / 3}\right)$. 
front originates from the quadratic $n$ dependence in $\partial_{x x} n^{2}$ [Eq. (5)]. In the context of our granular model this reflects that particles diffuse to neighboring boxes only through two-particle collisions: In the strong shaking limit the absence of particles slows down further diffusion, and the presence enhances it.

For nonzero $\tilde{B}$ [regime (ii), where the diffusive stage has to wait until after the sudden death] we have an additional dimensionless variable $\chi=\tilde{B} / C(t-\tau)$. Its influence diminishes with time and the solutions converge to the inverted parabola of the case $\tilde{B} \rightarrow 0$.

Next we turn to the second, semicontinuous stage. For moderate shaking, $\tilde{B} \approx \tilde{B}_{c, N}$, this stage can take quite a long time. At the critical point $\tilde{B}_{c, N}$ the lifetime $\tau$ even diverges to infinity and the cluster becomes stable. Starting out from the initial $\{\ldots, 0,0,1,0,0, \ldots\}$ distribution, the system first very slowly approaches a distribution in which $n_{c l}$ is close to the cluster density at the saddle-node bifurcation (see Fig. 2c) and all other boxes contain equal fractions $n_{k}=\left(1-n_{c l}\right) /(N-1)$. Only when it has passed this phantom equilibrium (i.e., when $n_{c l}$ is below the level of the saddle-node bifurcation), the system quickens its pace and the sudden collapse occurs.

This means that $\tau$ is the time it takes to pass the phantom equilibrium. It can be calculated either numerically or analytically [by integrating the Taylor expansion of Eq. (3)], with the result $\tau \propto\left(\tilde{B}_{c, N}-\tilde{B}\right)^{-1 / 2}$. So $\tau$ diverges as the inverse square root of the distance to the critical point, which is the common (mean field) power-law behavior near a second order phase transition as we have here for $\tau$ [8].

Finally, we calculate the lifetime away from the critical point, i.e., for $\tilde{B}$ considerably smaller than $\tilde{B}_{c, N}$. This will give us an analytical expression for the envelope curve in Fig. 2a. For these $\tilde{B}$ values, the collapse occurs before the particles leaking out of the cluster have had time to fill the outermost boxes to any significant level. Therefore, the behavior does not depend on the value of $N$ : The system does not feel its finite size during the cluster's lifetime, so the number of boxes can be taken to be infinite.

The time evolution of the cluster is described by Eq. (3), with $F\left(n_{-1}\right)=F\left(n_{1}\right)$ :

$$
\frac{d n_{c l}}{d t}=-2 F\left(n_{c l}\right)+2 F\left(n_{1}\right)=-2 F\left(n_{0}\right)+2 F\left(n_{1}\right) .
$$

This equation contains $n_{1}$, which is governed by a similar equation of motion [Eq. (3)] containing $n_{2}$, etc. So we have to deal with an infinite number of coupled nonlinear ODE's [9]. This is a problem that cannot be solved directly, so we attack it in five steps.

Step 1.-We first rewrite the problem into its continuum version, and replace the cluster density $n_{c l}$ by its lowdensity counterpart $n(0, t) \equiv n_{0}(t) \approx n_{c l} \exp \left(-\tilde{B} n_{c l}^{2} / 2\right)$. Thus, without influencing the fluxes [since $F\left(n_{0}\right)=$ $\left.F\left(n_{c l}\right)\right]$ we make $n(x, t)$ continuous in $x=0$. The density $n(x, t)$ obeys Eq. (4), plus a conservation condition saying that the increase of material into the rest of the system equals the influx from $x=0$ :

$$
\partial_{t} \int_{0}^{\infty} n(x, t) d x=-\partial_{x}(F(n(x, t)))_{x=0} .
$$

Step 2.-The cluster-equation Eq. (7) now becomes

$$
\begin{aligned}
\frac{d n_{c l}}{d t} & =2\left(\partial_{x} F(n(x, t))\right)_{x=0} \\
& =2 F^{\prime}(n(0, t))\left(\partial_{x} n(x, t)\right)_{x=0} .
\end{aligned}
$$

Since $F^{\prime}$ can be derived directly from Eq. (1), the problem reduces to determining $\partial_{x} n(x, t)$ at $x=0$.

Step 3.- In order to do so, we observe that changes in $n(0, t)$ happen on a much longer time scale than in the surrounding boxes [10], so the cluster acts as a constant reservoir spilling granular material. This approximation is illustrated in Fig. 4a: the profile in the system builds up while $n_{0}$ remains constant. In fact, this buildup takes place in a self-similar way (see Fig. 4b). So, as before, the problem for $n(x, t)$ can be formulated in terms of one variable $\xi=x /\left(n_{0} C \tilde{B}^{1 / 2} t\right)^{1 / 2}$. Setting $n(x, t)=n_{0} G(\xi)$, Eq. (4) becomes an ODE for $G(\xi)$, and also the accompanying conservation condition [Eq. (8)] contains $\xi$ only:

$$
\begin{aligned}
\frac{1}{2} \xi \partial_{\xi} G & =-\partial_{\xi \xi}\left(G^{2} e^{-\beta G^{2}}\right), \\
\left(\partial_{\xi} G\right)_{\xi=0} & =-\frac{e^{\beta}}{4(1-\beta)} \int_{0}^{\infty} G(\xi) d \xi,
\end{aligned}
$$

where $\beta=\tilde{B} n_{0}^{2}$.

Step 4.-The slope $\left(\partial_{x} n(x, t)\right)_{x=0}$ can now be approximated by $n_{0}(t)\left(\partial_{\xi} G(\xi)\right)_{\xi=0}\left(\partial_{x} \xi\right)_{x=0}$, where we have revived the (slow) time dependence in $n_{0}(t)$. With $\partial_{x} \xi=$ $\left(n_{0}(t) C \tilde{B}^{1 / 2} t\right)^{-1 / 2}$, Eq. (9) becomes

$$
\frac{d n_{c l}}{d t}=2 \frac{\sqrt{n_{0}(t)}}{\sqrt{C \tilde{B}^{1 / 2} t}} F^{\prime}\left(n_{0}(t)\right)\left(\partial_{\xi} G(\xi)\right)_{\xi=0} .
$$

All quantities on the right hand side are tractable. We reexpress $n_{0}(t)$ in terms of $n_{c l}(t)$, derive $F^{\prime}$ from Eq. (1), and solve Eq. (10) to determine $\left(\partial_{\xi} G(\xi)\right)_{\xi=0}$. This last step still requires some work, because Eq. (10) does not allow an analytical solution and moreover contains $\beta$ [and
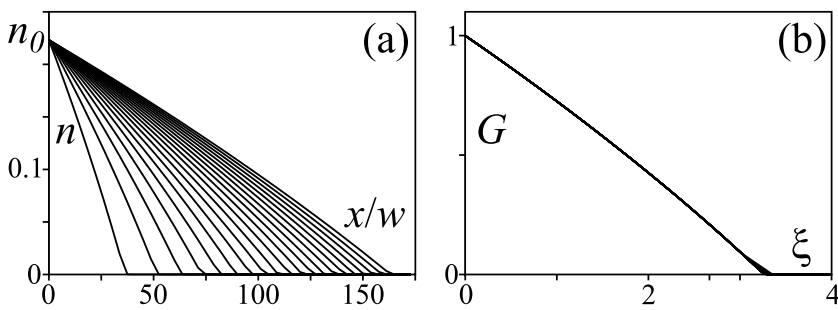

FIG. 4. (a) The density profile in the boxes surrounding the cluster, at 20 consecutive (equidistant) moments in time, within the constant $n_{0}$ approximation; $n_{0}=0.223$ and $\tilde{B}=3.00$. (b) The function $G=n / n_{0}$ onto which all curves in plot (a) collapse by a proper rescaling of the axes $\left(\xi \propto x t^{-1 / 2}\right)$. 
hence $n_{0}(t)$ ] explicitly. Since $\beta$ is small, however, we may expand $G(\xi)$ and Eq. (10) in terms of $\beta$, and solve numerically. In leading order we find $\left(\partial_{\xi} G(\xi)\right)_{\xi=0}=-K=$ -0.3138 . Inserting all this, Eq. (11) becomes

$$
\frac{d n_{c l}}{d t}=-4 \frac{K n_{c l}^{3 / 2} e^{-(3 / 4) \tilde{B} n_{c l}^{2}}}{\sqrt{C \tilde{B}^{1 / 2} t}}\left(1-\tilde{B} n_{c l}^{2} e^{-\tilde{B} n_{c l}^{2}}\right) \text {. }
$$

This is an ODE for $n_{c l}$ in closed form, which replaces the original problem [Eq. (7)] consisting of an infinite number of coupled ODE's.

Step 5.-Finally, we integrate Eq. (12) over the cluster density (dropping the suffix $c l$ ) and find an analytical expression for the lifetime $\tau$ away from the critical points:

$$
\tau=\left[\int_{n_{\mathrm{thr}}}^{1} \frac{\sqrt{C \tilde{B}^{1 / 2}} n^{-3 / 2} e^{(3 / 4) \tilde{B} n^{2}} d n}{8 K\left(1-\tilde{B} n^{2} e^{-\tilde{B} n^{2}}\right)}\right]^{2} .
$$

Here $n_{\text {thr }}$ is the value of $n$ at which the sudden death occurs. For the evaluation of the solid curve in Fig. 2a we used $n_{\text {thr }}=0.5$, but this value is not too critical (cf. Fig. 1e). The only free parameter is the constant $C$ : if this is adjusted properly, the analytical $\tau$ curve agrees with the measured data over the whole range of $\tilde{B}$ values.

The above expression shows that $\tau$ roughly increases as $\exp \left(\tilde{B}^{3 / 2}\right)$. Recalling that $\tilde{B}$ is the inverse shaking strength, this underlines the experimental observation that even a small reduction in the shaking strength causes a tremendous increase of the cluster lifetime.

In conclusion, in the studied compartmentalized system clusters break down very abruptly, in contrast to their slow formation. As clustering itself, the lack of time reversibility originates from the dissipative nature of the particle collisions: The breakdown of the unstable cluster is delayed because most of the energy input is dissipated through collisions in the cluster. The dynamics is quantitatively described by a remarkably simple flux model, which can be analytically solved.
We thank Ernst van Nierop for measuring the experimental lifetimes. This work is part of the research program of the Stichting FOM, which is financially supported by NWO.

[1] I. Goldhirsch and G. Zanetti, Phys. Rev. Lett. 70, 1619 (1993); S. McNamara and W. Young, Phys. Rev. E 50, R28 (1994); Y. Du, H. Li, and L. Kadanoff, Phys. Rev. Lett. 74, 1268 (1995); H. Jaeger, S. Nagel, and R. Behringer, Rev. Mod. Phys. 68, 1259 (1996); A. Kudrolli, M. Wolpert, and J. Gollub, Phys. Rev. Lett. 78, 1383 (1997); L. Kadanoff, Rev. Mod. Phys. 71, 435 (1999).

[2] J. Eggers, Phys. Rev. Lett. 83, 5322 (1999).

[3] K. van der Weele, D. van der Meer, M. Versluis, and D. Lohse, Europhys. Lett. 53, 328 (2001).

[4] D. van der Meer, K. van der Weele, and D. Lohse, Phys. Rev. E 63, 061304 (2001).

[5] Statistical fluctuations, which would add a noise term to Eq. (3), are left out of this (mean field) description.

[6] G. Barenblatt, Scaling, Self-Similarity, and Intermediate Asymptotics (Cambridge University Press, New York, 1996).

[7] G. Barenblatt, M. Bertsch, A. Chertock, and V. Prostokishin, Proc. Natl. Acad. Sci. U.S.A. 97, 9844 (2000); T. Witelski and A. Bernoff, Stud. Appl. Math. 100, 153 (1998).

[8] L. Landau and E. Lifshitz, Statistical Physics, Part 1 (Pergamon, Oxford, 1980), 3rd ed.

[9] The case $N=3$ is contained in a single ODE, which can be solved exactly: $\tau=\int_{n_{\mathrm{thr}}}^{1} d n / 2\{F[(1-n) / 2]-F(n)\}$ (solid curve in Fig. 2), with $n_{\text {thr }}$ determined from $\ddot{n}(\tau)=$ 0 . An analogous expression holds for general $N$ close to the critical point, where all the surrounding boxes are filled to the same level $n_{k}=\left(1-n_{c l}\right) /(N-1)$.

[10] A cluster change $\Delta n_{c l}$ (flowing into the surrounding boxes) corresponds to a much smaller change $\Delta n_{0}$ in its lowdensity counterpart: $d n_{c l} / d n_{0}=\left(1-\tilde{B} n_{c l}^{2}\right)^{-1} e^{\tilde{B} n_{c l}^{2} / 2} \approx$ $(1-\tilde{B})^{-1} e^{\tilde{B} / 2}$ since $n_{c l} \approx 1$. So for most $\tilde{B}$ values of interest (excluding the strong-shaking regime $\tilde{B} \rightarrow 0$ ) $\left|\Delta n_{c l}\right|=\sum_{i \neq 0} \Delta n_{i} \gg \Delta n_{0}$. 